\title{
一种梁式管接头密封性能与结构参数敏感性分析
}

\author{
崔 颖 ${ }^{1}$ 于预嘉 ${ }^{1}$ 王永亮 $^{1}$ 赵 爽 $^{2}$ 钟兢军 $^{3}$ \\ (1. 大连海事大学船舶与海洋工程学院 大连 116026; \\ 2. 中国航空发动机集团沈阳发动机研究所 沈阳 110015; \\ 3. 上海海事大学商船学院 上海 201306)
}

\begin{abstract}
摘要: 梁式管接头具有耐高压、自紧性和自封性等优点, 在航空液压系统中有着广阔的应用前景。设计提出了一种带有椭圆 形凹槽的梁式管接头阴接头结构, 建立了该型梁式管接头的弹塑性有限元接触模型, 数值模拟得到该结构能够形成两道有效 密封。以最大接触压力为密封性能评价指标的分析表明, 管内流体压力具有提高梁式管接头密封性能的作用, 且施加的预紧 力存在一个最佳设计值。该型梁式管接头阴、阳接头配合面在不同预紧力下的接触带宽试验结果与数值模拟结果吻合较好, 验证了有限元模型和分析方法的有效性。进而, 采用正交试验对梁式管接头的主要结构参数进行分析, 利用极差法得到了结 构参数对两道密封带密封性能影响的敏感性。结果表明, 椭圆形凹槽的长轴和短轴, 第一道密封的名义接触带宽与 U 形梁截 面宽度对带有椭圆形凹槽的梁式管接头密封性能有显著影响。
\end{abstract}

关键词: 梁式管接头; 弹塑性有限元; 密封性能; 敏感性; 正交试验

中图分类号: V231

\section{Sealing Performance and Sensitivity Analysis of Structure Parameters for a New Beam Seal}

\author{
CUI Ying ${ }^{1}$ YU Yingjia $^{1} \quad$ WANG Yongliang $^{1} \quad$ ZHAO Shuang $^{2} \quad$ ZHONG Jingjun $^{3}$
}

(1. College of Naval Architecture and Ocean Engineering, Dalian Maritime University, Dalian 116026;

2. Shenyang Engine Design Institute, Aero Engine Corporation of China, Shenyang 110015;

3. College of Merchant Marine, Shanghai Maritime University, Shanghai 201306)

\begin{abstract}
The beam seal has a broad application prospect in the aviation hydraulic system due to its high-pressure resistance, self-tightening and self-sealing features. A type of female joint structure with elliptical groove is proposed. The elastic-plastic finite element contact model of the beam seal is established. The analysis with the maximum contact pressure as the evaluation index of sealing performance shows that the fluid pressure in the pipe can improve the sealing performance of the beam seal, and there is an optimal design value for the applied pre-tightening force. The experimental results of the contact bandwidth on the mating surfaces of the female and male joints under different pre-tightening force are in good agreement with the numerical simulation, which verifies the effectiveness of the finite element model and analysis method. Furthermore, the main structural parameters of the beam seal are analyzed by orthogonal array method, and the sensitivity of the structural parameters to the sealing performance of the two sealing is obtained by the range method. It is found that the long and short axis of the elliptical groove, the nominal contact bandwidth of the first seal and the section width of the U-shaped beam have significant influence on the sealing performance of the beam seal.
\end{abstract}

Key words: beam seal; elastic-plastic finite element; sealing performance; sensitivity; orthogonal array method

\section{0 前言}

管路接头在航空航天等领域的液压系统中应用
广，数量多，是影响管路密封性能的重要元件。目 前我国航空液压管接头主要采用可分离的 $74^{\circ}$ 雉 形和球形密封结构 ${ }^{[1]}$ 。实践表明, 在管内流体压力 高于 $22 \mathrm{MPa}$ 的情况下现役结构多次出现泄漏问题,

\footnotetext{
* 教育部人文社科(18YJA880010)和国家自然科学基金青年基金(51606023) 资助项目。20200321 收到初稿, 20200927 收到修改稿
} 
其密封性能与可靠性叒待提高。

在各种类型管接头密封性能研究中有限元法被 广泛采用。康泽天等 ${ }^{[2]}$ 基于 ABAQUS 有限元分析软 件, 建立了形状记忆合金管接头与被连接管的光滑 无摩擦有限元模型, 分析了管接头几何结构参数与 扩径压力对密封接触压力的影响; 王小刚等 ${ }^{[3]}$ 利用 ANSYS 软件对雉头-雉孔、球头-雉孔两种雉形管接 头密封结构进行对比分析, 得到了两种结构的密封 性能; 韩冲等 ${ }^{[4]}$ 以 $60^{\circ}$ 扩口式球头-雉面密封结构为 研究对象, 采用有限元方法分析了密封面上的接触 压力与接触带宽随拧紧力矩的变化规律; 翟富刚 等 ${ }^{[5]}$ 针对一种弹性球头型管接头, 以接触压力和接 触带宽为密封性能的评判标准, 分析了工作压力下 管接头结构参数对密封性能的影响。这些研究表明, 基于传统管接头型式的改进结构在密封性能上难有 显著提高。

梁式管接头是一种兴起于美国的新型耐高压密 封型式, U 形梁使其具有自紧功能, 密封压力可达 $55 \mathrm{MPa}$, 并且梁式管接头具有安装插入尺寸短, 装 配维护方便的特点, 有望成为我国未来替代现役管 接头的密封型式 ${ }^{[6]}$ 。SAE 标准 ${ }^{[7-8]}$ 指出梁式管接头的 主要密封结构为阴接头的 U 形截面结构和密封梁。 梁式管接头与传统管接头的主要区别在于, 阴接头 密封面存在凹槽将其分成两部分, 在装配过程中形 成两道密封。然而, SAE 标准仅对梁式管密封阳接 头提出了尺寸要求, 对最为关键的阴接头未给出具 体结构参数。由于梁式密封具有弹塑性变形、两 道接触密封的特点, 设计研制难度大, 目前国内 外学者对梁式管接头的密封结构与性能研究仍处 于起步阶段。JEON 等 ${ }^{[9]}$ 和李晓东 ${ }^{[10]}$ 将 $U$ 形梁设 计为双雉度, 并应用有限元法研究了 $U$ 形梁雉角 与密封厚度对最大接触压力的影响, 该结构可在 密封面上形成较高的接触压力, 但未能形成两道 密封。目前梁式管接头构型设计和密封机理仍有 待进一步研究。

本文通过分析梁式管接头密封原理, 设计提 出了一种带有椭圆弧凹槽的梁式管密封阴接头结 构。建立该型梁式管接头的弹塑性有限元接触模 型, 数值模拟分析了两道密封的形成机理, 并试 验研究了该型密封结构的接触带宽与预紧力的关 系, 验证了理论分析的有效性。以最大接触压力 作为密封性能评判标准, 采用正交试验设计法分 析了主要结构参数对密封性能影响的敏感性, 以 期为梁式管接头结构设计和参数优化提供理论 指导。

\section{1 梁式管接头阴接头结构设计}

梁式管接头与唇式密封垫圈均要求具有自紧性 和高密封可靠性, 两者在结构上有诸多相似之处。 YANG 和 SALANT 等 ${ }^{[11-13]}$ 对往复轴密封中带有凹槽 的唇式 $U$ 形橡胶垫圈试验研究表明, 在 $U$ 形垫圈处 设计凹槽结构可在密封面形成二道密封, 使其密封 性能显著提高。借鉴 $U$ 形橡胶垫圈的凹槽结构, 以 AS4207A 的梁式管接头结构形式为基础, 设计一种 带有椭圆弧凹槽的梁式管接头, 如图 1 所示, 该结 构主要由阴、阳接头和外套螺母组成, 通过外套螺 母施加轴向预紧力, 在阴接头与阳接头接触面产生 接触压力, 对管内流体进行密封。

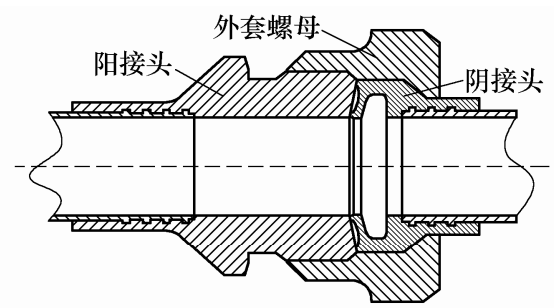

(a) 梁式管接头剖面图

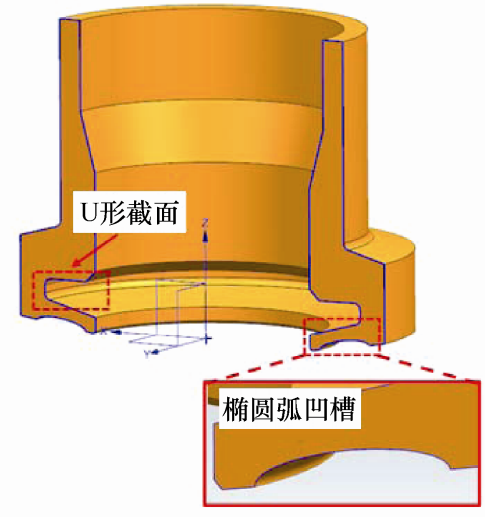

(b) 阴接头U形截面与椭圆弧山槽

图 1 梁式管接头结构图

针对该结构, 对带有椭圆形凹槽的阴接头进行 了详细的参数化设计, 其截面形状与结构参数如 图 2 所示, 其中: $d$ 为阳接头壁厚; $\alpha$ 代表阴接头密 封配合面与径向的夹角, 为锥面角; $h$ 为密封梁截 面宽度, $\theta$ 为 $\mathrm{U}$ 形梁与径向的夹角; $a$ 与 $b$ 分别为 $\mathrm{U}$ 形梁椭圆弧凹槽的长轴与短轴, $l$ 为沿阴接头雉面外 径与椭圆中心的距离, 控制椭圆弧的位置; $p$ 为椭 圆长轴与阴接头雉面间距离; $c$ 为第一道密封的名 义带宽; $\beta$ 与 $\gamma$ 为倒角角度; $R_{1}$ 与 $R_{2}$ 为倒圆角半径; $e$ 为 $\mathrm{U}$ 形口轴向长度, $\delta$ 为 $\mathrm{U}$ 形梁底部与阴接头外 径的距离。 


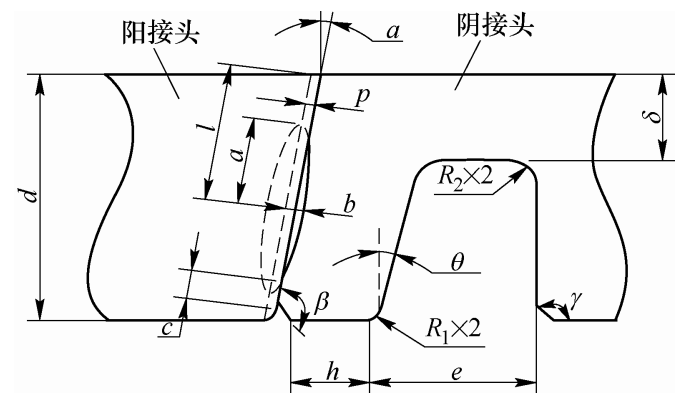

图 2 阴接头结构参数

\section{2 有限元建模与密封机理分析}

\section{1 有限元模型建立与约束条件设定}

为探究该型梁式管接头的密封机理, 预测其密 封性能, 利用有限元法建立梁式管接头弹塑性接触 模型。针对管径规格 $12 \mathrm{~mm}$, 阳接头结构尺寸根据 SAEMA2274 确定, 雉面角为 $8.5^{\circ}$, 壁厚为 $3.19 \mathrm{~mm}$ 。阴接头结构参数取值如表 1 所示。

\section{表 1 阴接头结构几何参数}

\begin{tabular}{|c|c|}
\hline 结构参数 & 取值 \\
\hline 阳接头壁厚 $d / \mathrm{mm}$ & 3.19 \\
\hline 凹槽的长轴 $a / \mathrm{mm}$ & 2.3 \\
\hline 凹槽的短轴 $b / \mathrm{mm}$ & 0.4 \\
\hline 沿阴接头雉面外径与椭圆中心的距离 $l / \mathrm{mm}$ & 1.67 \\
\hline 第一道密封的名义带宽 $c / \mathrm{mm}$ & 0.27 \\
\hline $\mathrm{U}$ 形梁底部与阴接头外径的距离 $\delta / \mathrm{mm}$ & 1.2 \\
\hline 椭圆长轴与阴接头雉面间距离 $p / m m$ & 0.1 \\
\hline $\mathrm{U}$ 形口轴向长度 $e / \mathrm{mm}$ & 1.8 \\
\hline $\mathrm{U}$ 形梁与径向的夹角 $\theta /\left(^{\circ}\right)$ & 11 \\
\hline 倒角角度 $\beta /\left(^{\circ}\right)$ & 126.5 \\
\hline 倒角角度 $\gamma /\left(^{\circ}\right)$ & 135 \\
\hline 密封梁截面宽度 $h / \mathrm{mm}$ & 0.6 \\
\hline 雉面角 $\alpha /\left(^{\circ}\right)$ & 8.5 \\
\hline 倒圆角半径 $R_{1} / \mathrm{mm}$ & 0.3 \\
\hline 倒圆角半径 $R_{2} / \mathrm{mm}$ & 0.1 \\
\hline
\end{tabular}

阴、阳接头材料均为不锈钢, 弹性模量 $E=210$ $\mathrm{GPa}$, 泊松比 $\mu=0.3$, 塑性本构关系参数如表 2 所示。 将阴接头接触面定义为主面, 阳接头接触面定义为 从面, 库伦摩擦系数设为 0.15 , 利用面面接触模型 和有限滑移公式对阴、阳接头接触面进行约束。在 外套螺母位置创建参考点 RP-1, 建立 RP-1 与阴接 头受力区域之间的分布耦合约束来模拟外套螺母与 管接头的连接关系, 如图 3 所示。
表 2 塑性本构关系参数设定

\begin{tabular}{cccc}
\hline 真实应力/MPa & 塑性应变 & 真实应力/MPa & 塑性应变 \\
\hline 500 & 0 & 882 & 0.25 \\
605 & 0.029 & 921 & 0.45 \\
695 & 0.056 & 988 & 0.75 \\
780 & 0.095 & 1040 & 0.85 \\
\hline
\end{tabular}

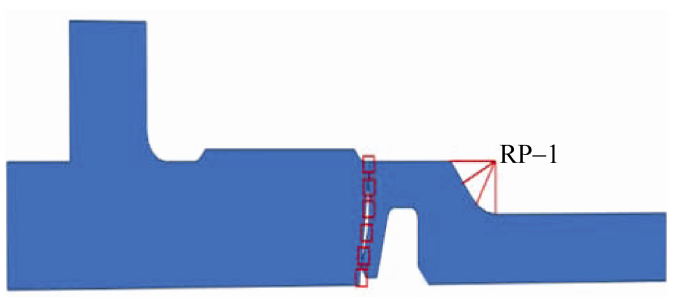

图 3 分布耦合约束与接触面

根据梁式管接头的实际受力情况, 在阳接头底 部施加固定约束, 在阳接头与外套螺母接触部分施 加径向位移约束, 如图 4 所示。在有限元计算过程 中, 通过对分布耦合约束参考点施加轴向位移荷载 来模拟预紧力的作用, 采用位移载荷是由于施加位 移载荷相比施加力载荷使求解过程更易于收敛。

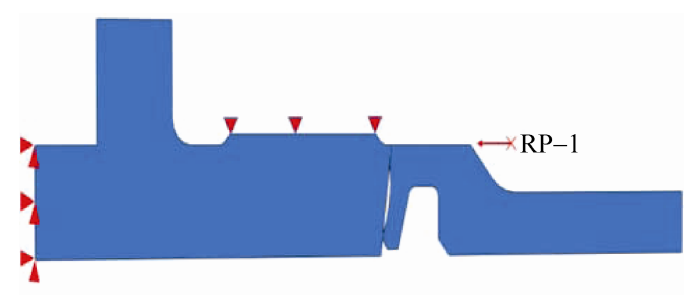

图 4 位移约束条件

有限元网格采用一阶非协调轴对称单元 CAX4I, 为防止主从面发生穿透, 对从面网格进行 加密, 阴接头网格尺寸为 $0.1 \mathrm{~mm}$, 阳接头网格尺寸 为 $0.05 \mathrm{~mm}$, 共划分网格 33741 个, 网格划分结果 如图 5 所示。采用 Newton-Raphson 法与对称罚函数 法来计算接触压力, 收玫准则为力和位移收玫准则。

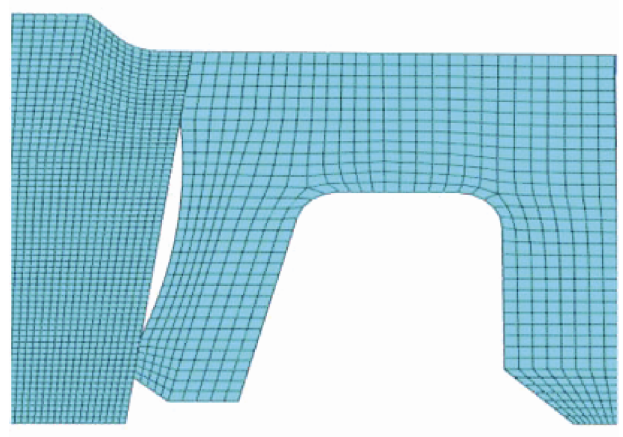

图 5 梁式管接头结构的网格划分 


\section{2 梁式管接头密封机理和性能分析}

阴、阳接头通过外套螺母施加的预紧力压紧, 对梁式管接头系统均匀施加轴向位移载荷 $0.1 \mathrm{~mm}$, 计算得到密封面压力分布云图, 如图 6 所示。可见, 在配合面上形成两道密封, 一道位于内径侧, 另一 道位于外径侧, 由于椭圆形凹槽的存在, 两道密封 之间形成密闭空腔, 构成隔离密封系统。当管内 通入高压流体时, 在内外压差的作用下流体经由 第一道密封的微观表面间隙向空腔内泄漏, 而空 腔内在装配过程中封闭了部分空气, 使流入空腔 的液体压力降低, 进而降低了第二道密封两侧的 压差, 从而可提高了管接头的密封性能。另外值 得注意的是, 第一道密封在靠近管内径处接触压 力为 0 , 这是由于外套螺母拧紧过程中, 可将密 封梁等效为一端受力的悬臂梁模型, 第二道密封 为固支端, 第一道密封的接触合力为密封梁施加 向上的支持力。在第一道密封接触合力的作用下， 密封梁内径端部发生翅曲, 使接触面分离, 导致 第一道密封内径处接触压力为零。管接头正常工 作时, 由于流体压力的作用, 在尧曲面上会形成 与密封接触压力相反的压力分布, 引起密封面接 触压力释放, 降低第一道密封的性能, 因此在设 计中需避免密封梁发生尧曲。

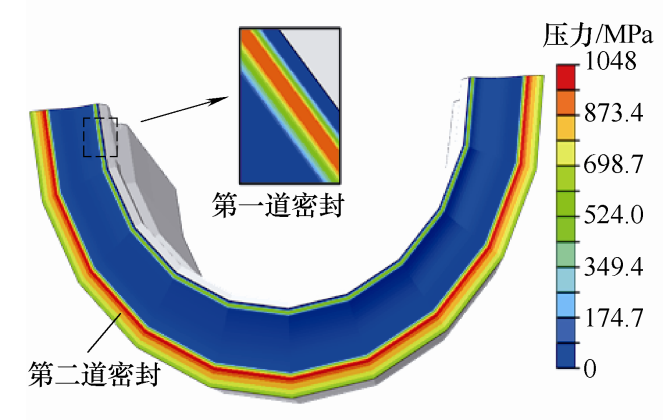

图 6 密封面接触压力分布云图

由阴、阳接头的等效塑性应变云图(图 7)与 Mises 应力云图(图 8)可知, 第一道密封为弹性变形,

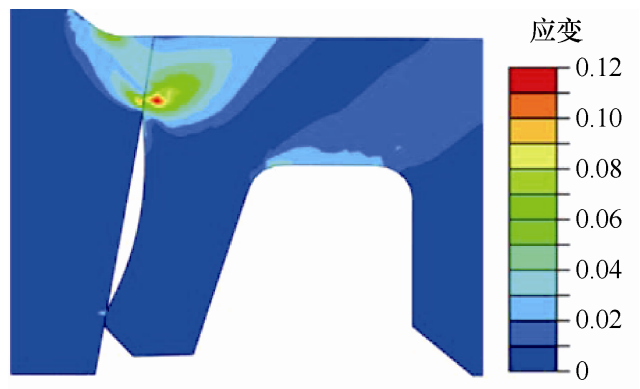

图 7 阴、阳接头的等效塑性应变云图

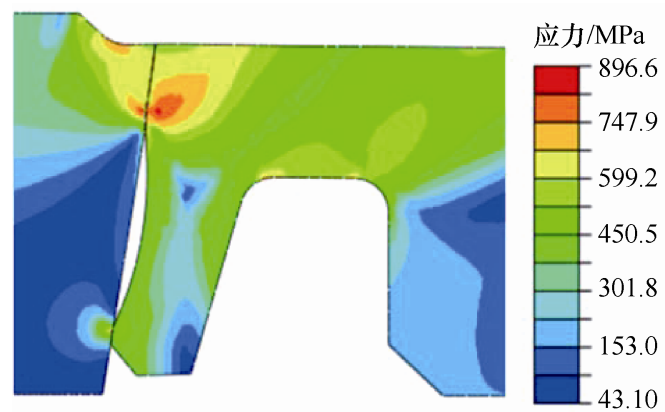

图 8 阴、阳接头的 Mises 应力云图

第二道密封为塑性变形, 密封梁为弹性变形。航 空管路连接处通常会受到较大的振动和弯曲力, 密封梁可防止振动和弯曲力传递到第一道密封。 而弹性密封梁会吸收管内的压力脉动, 防止压力 脉冲传递到第二道密封, 从而保证密封的稳定 可靠。

对于静密封结构, 增大接触压力会减小粗粘表 面接触产生的微观泄漏通道的截面面积和长度, 从 而提高密封性能 ${ }^{[14]}$ 。图 9 为对梁式管接头分别施加 $0.1 \mathrm{~mm}, 0.2 \mathrm{~mm}$ 和 $0.3 \mathrm{~mm}$ 的位移载荷, 得到不同 预紧力下密封面接触压力沿阳接头雉面母线的分布 曲线。可见, 随预紧力的增加, 第一道密封的最大 接触压力呈现先增大后减小的变化趋势, 过大的预 紧力会导致第一道密封失效。接触压力由式(1)计算 得到。

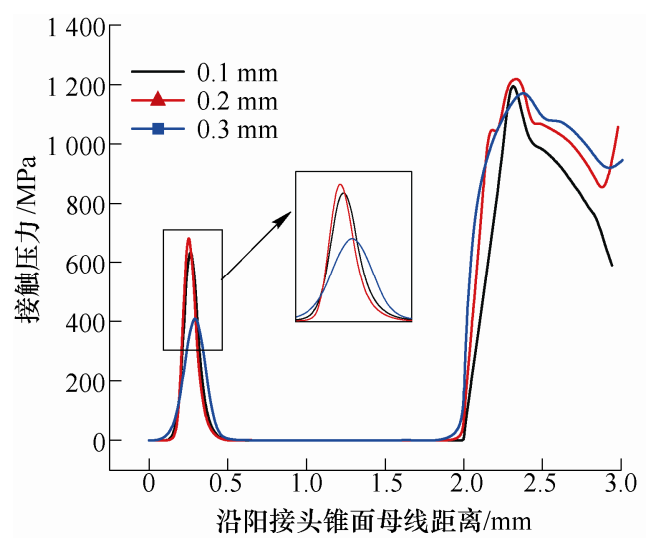

图 9 阴、阳接头配合面接触压力分布

$$
p=\frac{F}{A}
$$

式中, $F$ 为载荷分配到第一道密封的接触合力, $A$ 为第一道密封的接触面积。在施加 $0.1 \sim 0.2 \mathrm{~mm}$ 的 位移载荷时, 载荷较小, 密封梁在第一道密封处产 生微小变形, 第一道密封处接触压力随载荷的增大 而增加。当载荷增加到 $0.3 \mathrm{~mm}$ 时, 密封梁变形明显, 在第一道密封处椭圆弧边缘与阳接头接触, 接触面 
积 $A$ 的变化速率大于接触合力 $F$ 的变化速率, 使第 一道密封处接触压力降低, 因而对于目前所设计的 阴接头存在一个轴向预紧力的最优值, 能够保证第 一道密封处具有较高的接触压力。由于第二道密封 为塑性变形, 其最大接触压力无明显变化, 预紧力 对第二道密封性能影响很小。因此, 第二道密封可 有效防止因螺母扭矩过大造成的第一处密封失效, 提高梁式管接头的可靠性。

图 10 为不同流体内压情况下, 第一道密封接触 压力沿阳接头雉面母线的压力分布曲线。可见, 最 大接触压力随流体内压的增加而增大, 这是由于流 体内压对 U 形区域施加了额外的压紧力导致的, 因 此高压流体可提高第一道密封的密封性能。流体压 力对第二道密封没有直接作用, 故第二道密封的密 封性能不随流体压力的提高而改变。因此, 该型梁 式管接头具有自封特性, 可用于工作压力较高的液 压系统。

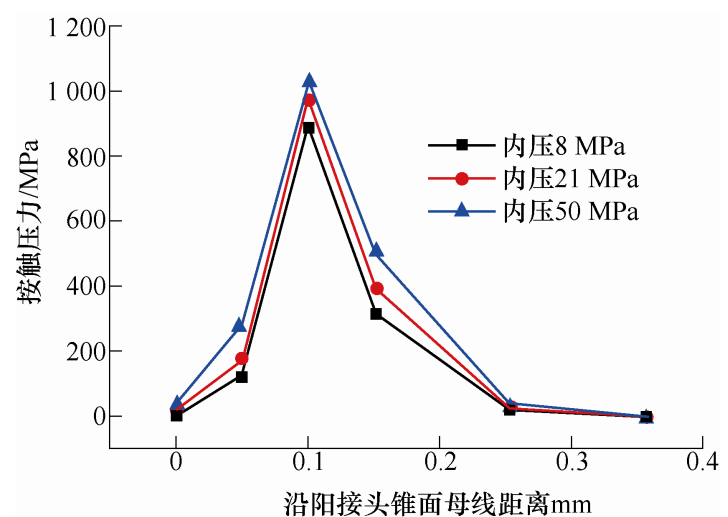

图 10 不同流体内压下第一道密封带接触压力分布

\section{3 梁式管接头密封接触试验}

\section{1 试验装置与方案}

为验证该型梁式管接头可形成两道密封与有 限元计算结果的正确性, 设计加工了阴、阳接头 结构, 采用如图 11 所示的试验装置开展密封带宽 的试验。试验由阳接头固定件、加力件、压力传 感器、阳接头、阴接头、垫片与液压缸组成。加 力件与阴接头配合模拟外套螺母对梁式管接头施 加预紧力, 阴接头、阳接头与加力件配合构成试 验主体。为准确测量密封面上接触压力分布与接 触带宽, 将高精度 FUJI 压敏测试胶片放置于阴阳 接头接触配合面, 如图 12 所示。垫片与加力件之 间安装压力传感器, 用于测量施加于梁式管接头 的预紧力。

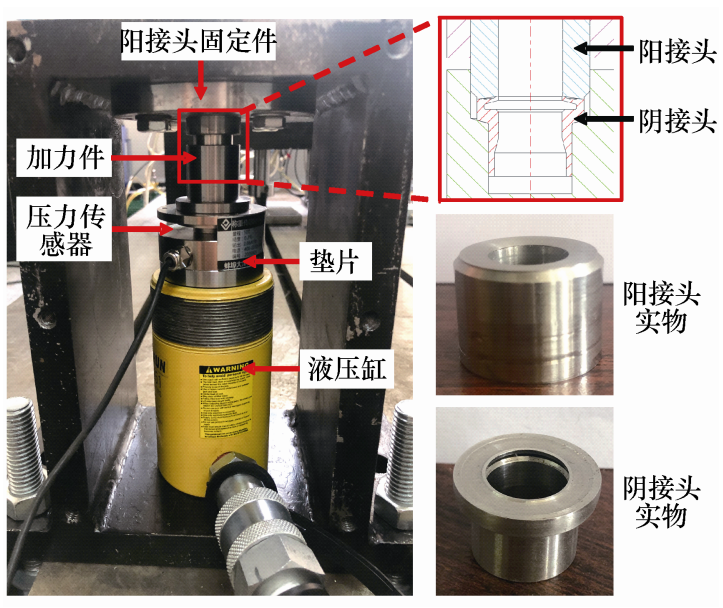

图 11 试验装置和试验件

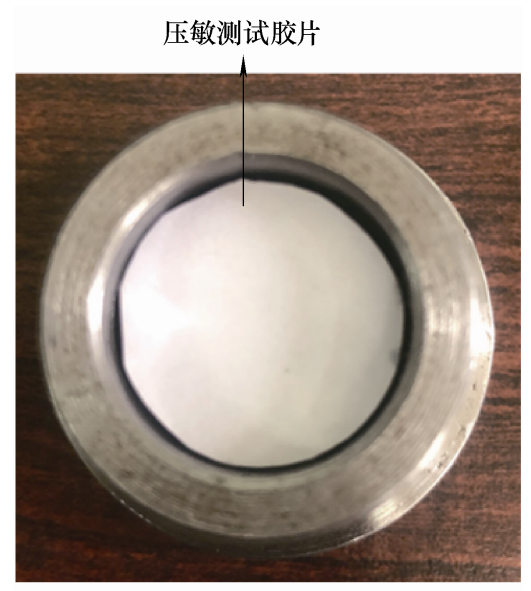

图 12 压敏测试胶片放置图

\section{2 试验结果分析}

施加 $1000 \sim 8000 \mathrm{~N}$ 预紧力, 观察压敏测试胶 片的颜色变化情况, 若胶片颜色较深, 则接触压力 大, 反之接触压力小, 粉色环状带的带宽代表梁式 管接头真实接触带宽。阴、阳接头配合面接触试验 的测量结果如图 13 所示。可观察到, 受试验件加工 误差与装配偏差的影响, 在预紧力低于 $6000 \mathrm{~N}$ 时 内圈的第一道密封未完全封闭形成有效密封, 随着 预紧力增大，压敏胶片上逐渐形成两道明显粉色环 状带, 表明该梁式管接头可形成两道密封。

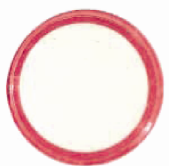

(a) $1000 \mathrm{~N}$

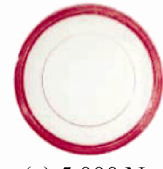

(e) $5000 \mathrm{~N}$

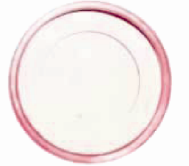

(b) $2000 \mathrm{~N}$

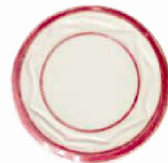

(f) $6000 \mathrm{~N}$

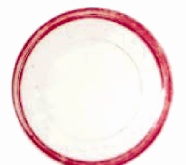

(c) $3000 \mathrm{~N}$

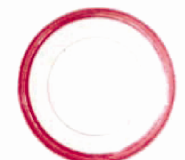

(g) $7000 \mathrm{~N}$

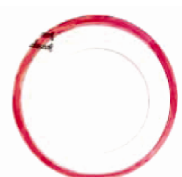

(d) $4000 \mathrm{~N}$

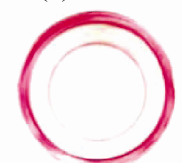

(h) $8000 \mathrm{~N}$
图 13 不同预紧力下压敏胶片显示结果 
应用 UG 软件对压敏胶片上的密封环带宽进行 光栅测量, 压敏测试胶片在受压时有少量颜色溢出, 因而在光栅测量接触带宽时以最小接触带宽为准, 如图 14 所示。

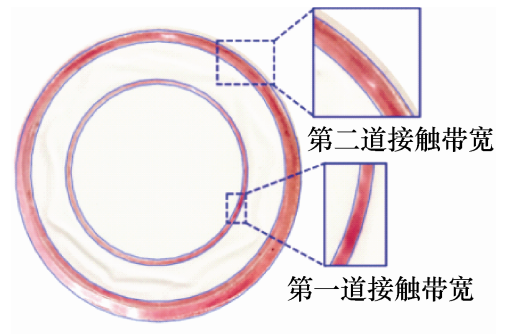

图 14 光栅测量接触带宽

将获得的不同预紧力下两道密封的接触带测量 结果与模型有限元计算进行对比, 如图 15 所示。试 验结果与有限元模拟结果趋势相同, 试验得到两道 密封的接触带宽均高于数值模拟结果, 这与压敏胶 片在受到较大压力时会有少量颜料渗出有关, 试验 结果与计算结果平均误差为 $20 \%$, 数据吻合较好, 验证了本文所采用的接触有限元分析方法的有效 性。进而, 由图 15a 可知, 第一道密封的名义带宽

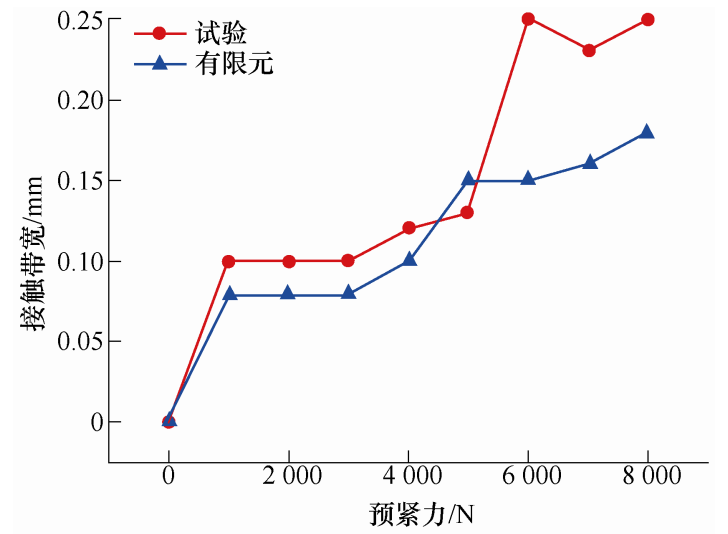

(a) 第一道密封接触带宽对比

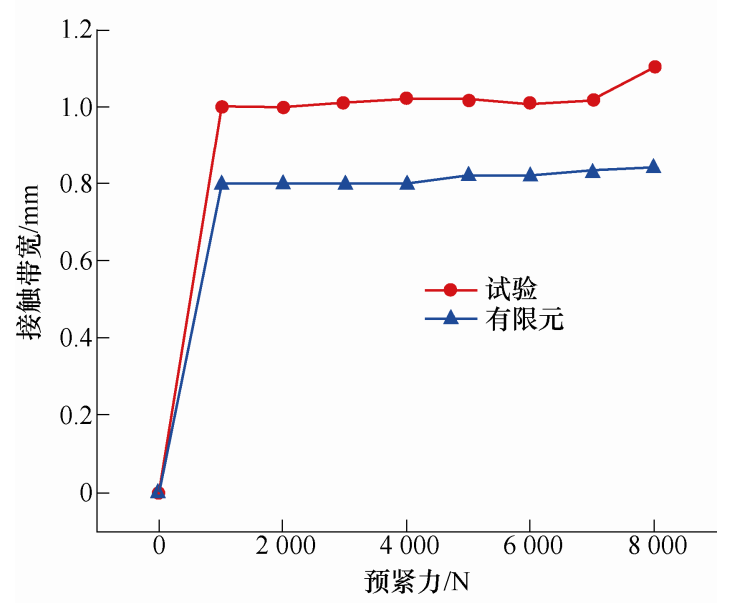

(b) 第二道密封接触带宽对比

图 15 两道密封试验结果与有限元计算结果对比
为 0.3 , 试验结果的带宽值均小于 0.3 , 说明梁式管 接头在压紧过程中, 内径端部会发生尧曲, 第一道 密封的主要密封区域在靠近椭圆弧凹槽侧; 图 15b 的试验结果表明, 第二道密封接触带宽对预紧力的 变化并不敏感, 这与数值模拟得到的结果一致。

\section{4 结构参数对梁式管接头密封性能的 敏感性分析}

国内外学者通常采用单一变量原则对管接头结 构参数进行分析, 但对于复杂的结构, 参数之间相 互牵连, 单独改变某一变量不能充分描述参数对目 标函数能带来的影响 ${ }^{[15]}$ 。

正交试验设计可同时改变所有结构参数并充分 描述参数对目标函数的影响, 本文以最大接触压力 为目标函数, 采用 $L_{27}\left(3^{13}\right)$ 正交表针对决定 U 形梁形 状的 6 个结构参数进行正交试验设计, 结构参数水 平如表 3 所示。

表 3 正交试验中的结构参数

\begin{tabular}{cccc}
\hline 结构参数 & \multicolumn{3}{c}{ 水平 } \\
\cline { 2 - 4 } & 1 & 2 & 3 \\
\hline 密封梁截面宽度 $h / \mathrm{mm}$ & 0.70 & 0.85 & 1.00 \\
第一道密封的名义带宽 $c / \mathrm{mm}$ & 0.20 & 0.35 & 0.50 \\
凹槽的长轴 $a / \mathrm{mm}$ & 1.50 & 1.75 & 2.00 \\
凹槽的短轴 $b / \mathrm{mm}$ & 0.2 & 0.3 & 0.4 \\
雉面角 $\alpha /\left(^{\circ}\right)$ & 0 & 5 & 10 \\
$\mathrm{U}$ 形梁与径向的夹角 $\theta /\left({ }^{\circ}\right)$ & 15.0 & 17.5 & 20.0 \\
\hline
\end{tabular}

27 组试验计算结果如图 16 所示, 图中 $y$ 轴表 示第一道密封与第二道密封的最大接触压力, $x$ 轴 表示试验组编号。各结构参数的效应值反映了结构 参数对第一道密封最大接触压力的影响关系 ${ }^{[16]}$, 效 应值 $F_{i}^{j}$ 的计算方法如下

$$
\begin{gathered}
\bar{F}=\frac{\sum_{i=1}^{27} F\left(X_{i}\right)}{27} \\
\bar{F}_{i}^{j}=\frac{1}{r} \sum_{F_{i}^{j} \in F\left(X_{i}\right)} F\left(X_{i}\right) \\
F_{i}^{j}=\bar{F}_{i}^{j}-\bar{F}
\end{gathered}
$$

式中, $F\left(X_{i}\right)$ 为各组试验最大接触压力值, $\bar{F}$ 为 27 组试验的数据平均值, $\bar{F}_{i}^{j}$ 为 $i$ 参数第 $j$ 水平的数据 平均值, $r$ 为正交表任一列上各水平出现的次数, $F_{i}^{j}$ 为 $i$ 参数 $j$ 水平的效应值。 


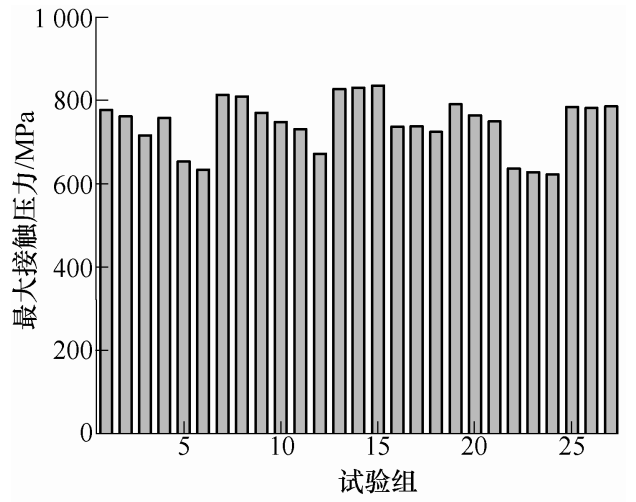

(a) 第一道密封最大接触压力

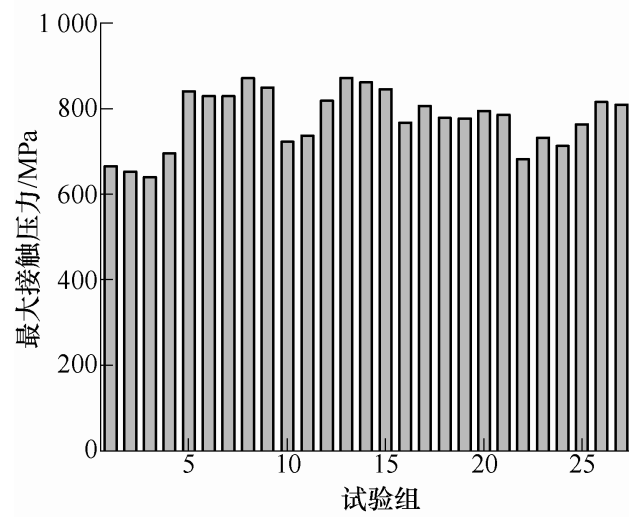

(b) 第二道密封最大接触压力

图 16 各组试验值

将图 16 中的计算结果代入式(2) (4), 得到各 结构参数的效应值计算结果如图 17、18 所示。若效 应值为正, 代表该参数的取值会使最大接触压力增 大, 若效应值为负, 则该参数的取值使最大接触压 力减小。由图 17、18 可知, 第一道密封最大接触压 力随第一道密封名义接触带宽 $c$ 与椭圆凹槽长轴 $a$ 呈现先减小后增大的变化趋势, 随阴接头雉面角 $\alpha$ 、 $\mathrm{U}$ 形梁与径向夹角 $\theta$ 、圆凹槽短轴 $b$ 的减小而降低, 随密封梁截面高度 $h$ 的增大而减小。第二道密封最 大接触压力随第一道密封名义接触宽度 $c$ 与椭圆凹 槽长轴 $a$ 的增大而增加, 随 $\mathrm{U}$ 形梁截面宽度 $h$ 、阴 接头雉面角 $\alpha 、 \mathrm{U}$ 形梁与径向夹角 $\theta$ 、圆凹槽短轴 $b$ 呈现先增大后减小的趋势。

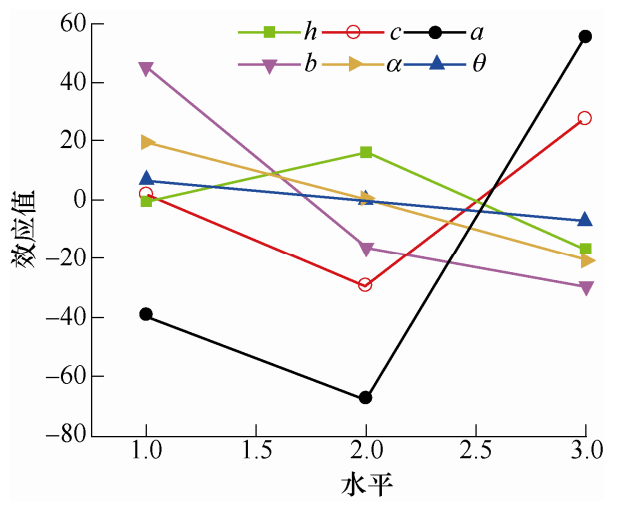

图 17 第一道密封结构参数效应值

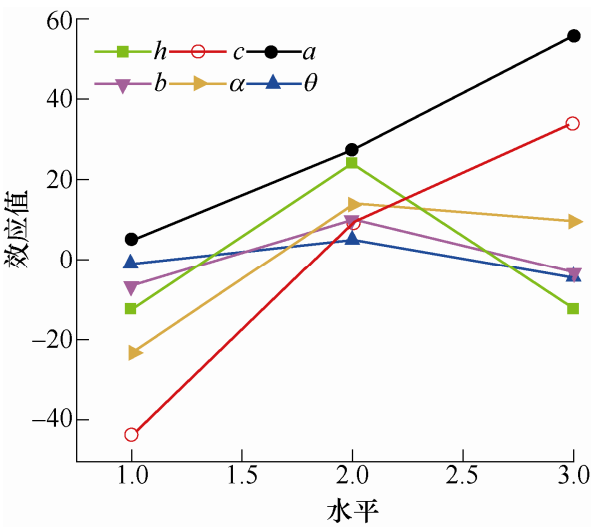

图 18 第二道密封结构参数效应值

为评价各结构参数对密封带最大接触压力的敏 感度, 采用正交试验极差分析法对其进行研究 ${ }^{[17]}$ 。 极差是反应参数对目标函数重要性的重要指标, 极 差 $R_{i}$ 的计算方法如下

$$
R_{i}=\max \left(\bar{F}_{i}^{j}\right)-\min \left(\bar{F}_{i}^{j}\right)
$$

将图 17 中的计算结果代入式(5), 经计算得到 极差 $R_{i}$, 极差越大，则表示 $i$ 参数对第一道密封的 最大接触压力的影响越大, 各参数的极差值计算结 果如图 19 所示。

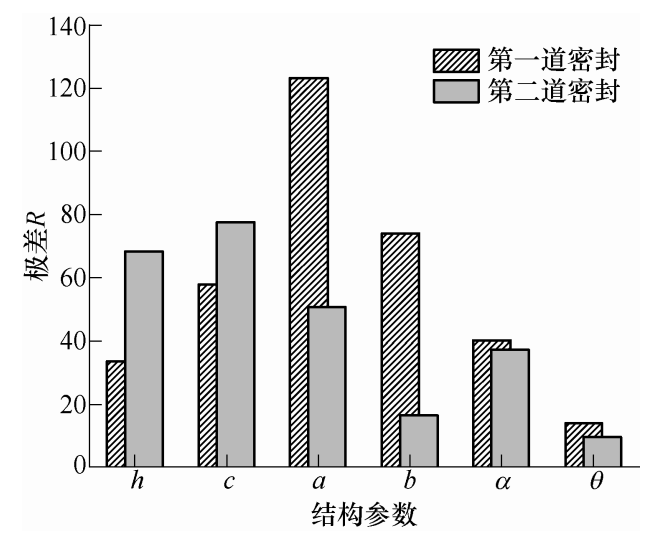

图 19 结构参数极差值

由图 19 可知, 各结构参数对第一道密封最大接 触压力的影响顺序为 $a>b>c>\alpha>h>\theta$, 由此可 见, 椭圆形凹槽的长轴 $a$, 短轴 $b$ 与第一道密封的 名义接触带宽 $c$ 对第一道密封的密封性能影响显 著。其原因是以上三个参数共同确定了两道密封的 形成位置和第二道密封的名义接触宽度, 进而影响 轴向预紧力在两道密封上压紧力的分配比例和两密 封面的接触压力分布。对于第二道密封, 结构参数 对最大接触压力影响程度排序为 $c>a>h>\alpha>b>\theta$ 。因此, 第一道密封名义接触带 宽 $c$, 椭圆凹槽长轴 $a$ 与 $\mathrm{U}$ 形梁截面宽度 $h$ 对第二 道密封的性能影响显著。综合考虑结构参数对两道 
密封最大接触压力的影响, 该型密封结构的设计中 需要着重针对 $a, b, c, h$ 四个结构参数进行优化。

\section{5 结论}

(1) 设计提出一种带有椭圆弧凹槽的梁式管接 头阴接头结构, 数值模拟和试验研究表明该管接头 结构可形成两道密封。第一道密封产生弹性接触, 第二道密封产生塑性接触。两道密封环之间部分相 当于弹性梁, 使密封具有可承受振动和高压脉冲的 自紧特性。

(2) 该型梁式管接头预紧力超过某一阀值时会 导致第一道密封失效, 因而预紧力存在一个最佳设 计值。第二道密封接触压力随预紧力变化相对稳定, 为第一道密封失效后提供了防止泄漏的安全保障。

(3) 管路内流体内压的增大会使得该型梁式管 接头的第一道密封环接触压力增加, 数值研究表明 该型梁式管接头可适用于 $50 \mathrm{MPa}$ 高压流体管路 系统。

（4）采用正交试验设计法对结构参数对密封性 能影响的敏感性分析表明, 椭圆形凹槽的长轴、短 轴、第一道密封的名义接触带宽与 U 形梁截面宽度 对该型梁式管接头密封性能有显著影响, 为进一步 的结构优化设计提供了参考依据。

\section{参 考 文 献}

[1] 付长安, 宋治国. 超高压飞机液压系统的密封问题[J]. 润滑与密封, 2000(4): 5-6.

FU Changan, SONG Zhiguo. Sealing problem of hydraulic system of ultra-high pressure aircraft[J]. Lubrication Engineering, 2000(4): 5-6.

[2] 康泽天, 周博, 薛世峰. 形状记忆合金管接头热机耦合 行为的有限单元法[J]. 机械工程学报, 2018, 54(18): 68-75.

KANG Zetian, ZHOU Bo, XUE Shifeng. Finite element numerical simulation on thermo-mechanical coupling behavior in shape memory alloy pipe connection[J]. Journal of Mechanical Engineering, 2018, 54(18): 68-75.

[3] 王小刚, 张方晓, 黄鹏. 基于 ANSYS 的管路雉形密封 结构参数分析 [J]. 兵工自动化, 2009, 28(8): 25-27. WANG Xiaogang, ZHANG Fangxiao, HUANG Peng. Analysis of parameters of pipeline cone sealing structures based on ANSYS[J]. Ordnance Industry Automation, 2009, 28(8): 25-27.

[4] 韩冲, 张勇. 导管连接件球头-雉面结构密封性能研
究[J]. 润滑与密封, 2012(10): 105-112.

HAN Chong, ZHANG Yong. Research on sealing property of globe-cone joint for pipeline[J]. Lubrication Engineering, 2012(10): 105-112.

[5] 翟富刚, 孙玉岗, 豆旭安, 等. 某超高压管接头结构参 数对接触密封性能的影响分析 [J]. 液压与气动, 2017(6): 60-64.

ZHAI Fugang, SUN Yugang, DOU Xuan, et al. Influence of structural parameters on sealing performance of ultra-high pressure pipe joint[J]. Chinese Hydraulics \& Pneumatics, 2017(6): 60-64.

[6] 欧阳小平, 方旭, 朱䒯, 等. 航空液压管接头综述 [J]. 中 国机械工程, 2015, 26(16): 2262-2271.

OUYANG Xiaoping, FANG Xu, ZHU Ying, et al. Overview of aviation hydraulic fitting[J]. China Mechanical Engineering, 2015, 26(16): 2262-2271.

[7] AS4207A Fitting End, External thread, beam seal, design standard[S]. Warrendale, PA: SAE, 1996.

[8] AS4209B Fitting End Assembly, internal thread, retained nut, beam seal, design standard[S]. Warrendale, PA :

SAE, 1997.

[9] JEON J Y, KIM B T. A study on contact characteristics by the geometry variation of beam seal fitting of an aircraft fuel hose[J]. Journal of the Korean Society of Manufacturing Process Engineers, 2013, 12(6): 101-108.

[10] 李晓东. 可分离式航空液压管接头密封特性研究 [D]. 大连: 大连理工大学, 2018.

LI Xiaodong. Sealing performance of separable aviation hydraulic fittings[D]. Dalian : Dalian University of Technology, 2018.

[11] SALANT R F, MASER N, YANG B. Numerical model of a reciprocating hydraulic rod seal[J]. Journal of Tribology, 2006, 129(1): 91-97.

[12] SHI Fanghui , SALANT R F . Numerical study of a rotary lip seal with a quasi-random sealing surface[J]. Journal of Tribology, 2001, 123(3): 517-525.

[13] YANG B, SALANT R F. A numerical model of a reciprocating rod seal with a secondary lip [J]. Tribology Transactions, 2008, 51(2): 119-127.

[14] 高连新, 金烨, 张居勤. 石油套管特殊螺纹接头的密封 设计[J]. 机械工程学报, 2005, 41(3): 216-220.

GAO Lianxi, JIN Ye, ZHANG Juqin. Seal design of premium threaded casing connections[J]. Journal of Mechanical Engineering, 2005, 41(3): 216-220.

[15] TAGUCHI G, CHOWDHURY S, WU Y. Taguchi's 
quality engineering handbook[M]. Hoboken: John Wiley, 2005.

[16] JIA Xiaohong, GUO Fei, SALANT R F, et al. Parameter analysis of the radial lip seal by orthogonal array method[J]. Tribology International，2013， 64: 96-102.

[17] HU Chaobin, ZHANG Xiaobing. Influence of multiple structural parameters on interior ballistics based on orthogonal test methods[J]. Defence Technology, 2019, 56(4): 201-208.

作者简介: 崔颖, 女, 1977 年出生, 博士, 副教授。主要研究方向为转 子动力学、管路连接结构密封接触力学。

E-mail: cuiying@dlmu.edu.cn

于颖嘉(通信作者), 男, 1990 年出生, 博士研究生。主要研究方向为金 属静密封接触力学与振动控制。

E-mail: cf_yuyingjia@sina.com 